\title{
Correction for: YKL40 in sporadic amyotrophic lateral sclerosis: cerebrospinal fluid levels as a prognosis marker of disease progression
}

\section{Pol Andrés-Benito ${ }^{1,2,3}$, Raúl Domínguez ${ }^{4}$, Maria J. Colomina ${ }^{5}$, Franc Llorens ${ }^{2,3}$, Mònica Povedano ${ }^{4}$, Isidre Ferrer ${ }^{1,2,3,6,7}$}

${ }^{1}$ Department of Pathology and Experimental Therapeutics, University of Barcelona, L'Hospitalet de Llobregat, Barcelona, Spain

${ }^{2}$ Biomedical Network Research Center on Neurodegenerative Diseases (CIBERNED), Institute Carlos III, L'Hospitalet de Llobregat, Barcelona, Spain

${ }^{3}$ Bellvitge Biomedical Research Institute (IDIBELL), L'Hospitalet de Llobregat, Barcelona, Spain

${ }^{4}$ Functional Unit of Amyotrophic Lateral Sclerosis (UFELA), Service of Neurology, Bellvitge University Hospital, L'Hospitalet de Llobregat, Barcelona, Spain

${ }^{5}$ Anesthesia and Critical Care Department, Bellvitge University Hospital - University of Barcelona L'Hospitalet de Llobregat, Barcelona, Spain

${ }^{6}$ Neuropathology, Pathologic Anatomy Service, Bellvitge University Hospital, IDIBELL, L'Hospitalet de Llobregat, Barcelona, Spain

${ }^{7}$ Institute of Neurosciences, University of Barcelona, Barcelona, Spain

Correspondence to: Zhao Di, Fan Ruitai; email: fcczhaod@zzu.edu.cn, fccfanrt@zzu.edu.cn

Original article: Aging (Albany NY) 2018; 10: pp $2367-2382$

PMID: $30215603 \quad$ PMCID: $\underline{\text { PMC6188478 } \quad \text { doi: } 10.18632 / a g i n g .101551}$

This article has been corrected: YKL40 values in text and figures represent $\mathbf{n g} / \mathbf{m L}$, not $\mathrm{pg} / \mathrm{mL}$ This alteration does not affect the results or conclusions of this work. 